\title{
REVISIÓN DE LA LITERATURA \\ SOBRE ECONOMÍA INFORMAL Y TRABAJO DE MUJERES*
}

\author{
Recibido: 13 de febrero de 2018 - Aprobado: 11 de agosto de 2018 \\ https://doi.org/10.22395/seec.v21n48a2
}

Mayda Alejandra Calderón Díaz ${ }^{* *}$

\section{RESUMEN}

Esta investigación presenta una revisión de la literatura sobre economía informal y trabajo de mujeres contemplada desde las visiones de autores de las escuelas estructuralista, voluntarista, legalista y dualista. El objetivo de este estudio es enunciar algunas de las diferentes posiciones doctrinales del concepto de la economía informal, la evolución del trabajo de mujeres y las críticas que se le han hecho al concepto de informalidad. Los autores mencionados en este trabajo son referentes actuales en la materia debido a que en su mayoría son los más citados. El estudio concluye con el argumento de que algunos de ellos entienden el fenómeno de la informalidad como un refugio involuntario y otros como una opción para evadir impuestos.

\section{PALABRAS CLAVE}

Economía informal; trabajo de mujeres; mercado de trabajo; informalidad.

\section{CLASIFICACIÓN JEL}

E20, E24, E26, J40

\section{CONTENIDO}

Introducción; 1. Trabajo de mujeres; 2. Concepto de informalidad; 3. Críticas al concepto de informalidad; 4. Análisis de la economía informal y trabajo informal de mujeres; 5 . Conclusiones; 6. Líneas futuras de investigación y debilidades del estudio; Bibliografía.

Revisión bibliográfica correspondiente a la tesis doctoral: "Mujeres y trabajo informal en la ciudad de Bogotá: una aproximación experimental".

* Economista, Universidad Industrial de Santander, Bucaramanga, Colombia. Magíster en Administración, Universidad Nacional de Colombia, Bogotá, Colombia. Candidato a doctor en Ciencias Económicas, Universidad Nacional de Colombia, Bogotá, Colombia. Investigadora interna y visitante académica, Middlesex University London, Londres, Reino Unido. Dirección: 27 Anson Road, Código postal: N7 ORB London. Correo electrónico: maacalderondi@unal.edu.co 


\title{
LITERATURE REVIEW ON INFORMAL ECONOMY AND WOMEN'S LABOR
}

\begin{abstract}
This research presents a literature review about informal economy and women's labor from structuralist, voluntarist, legalist and dualist schools' authors. The objective is to enunciate some of the different doctrinal positions of the informal economy concept, the evolution of women's labor and criticisms made to the concept of informality. The authors mentioned in this paper are current references in the matter because they are the most cited. The study concludes that some of them understand the phenomenon of informality as an involuntary refuge and others as an option to evade taxes.
\end{abstract}

\section{KEYWORDS}

Informal economy; women's labor; labor market; informality.

\section{JEL CLASSIFICATION \\ E20, E24, E26, J40}

\section{CONTENT}

Introduction; 1 . Women's labor; 2. Concept of informality; 3 . Criticisms made to the informality concept; 4. Analysis of the informal economy and women's informal labor; 5 . Conclusions; 6 . Future research lines and the study's weaknesses; Bibliography.

\section{REVISÃO DA LITERATURA SOBRE ECONOMIA INFORMAL E TRABALHO DE MULHERES}

\section{RESUMO}

Esta pesquisa apresenta uma revisão da literatura sobre economia informal e trabalho de mulheres a partir da visão de autores das escolas estruturalista, voluntarista, legalista e dualista. O objetivo deste estudo é enunciar algumas das diferentes posições doutrinais do conceito de economia informal, evolução do trabalho de mulheres e críticas que são feitas ao conceito de informalidade. Os autores mencionados neste trabalho são referentes atuais na matéria devido a que, em sua maioria, são os mais citados. Este estudo conclui com o argumento de que alguns deles entendem o fenômeno da informalidade como um refúgio involuntário, e outros, como uma opção para sonegar impostos.

\section{PALAVRAS-CHAVE}

Economia informal; trabalho de mulheres; mercado de trabalho; informalidade.

\section{CLASSIFICAÇÃO JEL}

E20, E24, E26, J40

\section{CONTEÚDO}

Introdução; 1 . Trabalho de mulheres; 2. Conceito de informalidade; 3 . Críticas ao conceito de informalidade; 4. Análise da economia informal e trabalho informal de mulheres;

5. Conclusões; 6 . Linhas futuras de pesquisa e debilidades do estudo; Bibliografia. 


\section{INTRODUCCIÓN}

Al revisar la literatura sobre los condicionantes de la pobreza en el caso de las mujeres, se encuentran obstáculos conceptuales y de medición. De acuerdo con Barbieri (1982) aparecen deficiencias en cuanto a la claridad y comprensión en la conceptualización de ciertas actividades donde la mujer se desempeña, como por ejemplo el hogar. De igual manera, la concepción de familia como monolítica, que dista del modelo real de familia actual. En segunda medida, la rígida caracterización o definición de trabajo, aun en el sector informal de la economía, a excepción de la multiplicidad de actividades que desempeñan las mujeres, sobre todo en sectores populares y campesinos, para garantizar la reproducción diaria de la vida en la unidad doméstica y cuyas variables no son consideradas como trabajo.

Es decir, así como lo considera Barquet (1991, p. 45) "se trata de la ausencia de valor atribuido a esas actividades en el mercado, sin valorar su aporte económico, derivada de la creencia que muchas actividades se atribuyen naturales a las mujeres. De la misma manera aparecen problemas de medición que se derivan, de manera directa, de los anteriores y ofrecen, por tanto, un panorama poco nítido de las condiciones de vida de amplios sectores de la población femenina".

Por lo tanto, Salles y Tuirán (1999) argumentan la existencia de una grave carencia de información sobre características específicas de la incidencia de determinados fenómenos en el caso de las mujeres, así como el registro de su participación en ellos. En algunas ocasiones, se encuentran con cifras que no desglosan la información entre hombres y mujeres o con análisis que no muestran la influencia de factores tan importantes para la participación económica de la mujer, como lo son la clase, la edad y la posición en el ciclo vital.

Este interés por investigar el trabajo informal de las mujeres surge a partir de los reportes de la Organización Internacional del Trabajo (OIT, 2002) en los que se estima que en el mundo la proporción de mujeres en el empleo informal urbano es del 74 \%. Según Rosales (2003, p. 25) "en Colombia la mayoría de los trabajadores informales son asalariados (62 \%) y de sexo femenino, los trabajadores de la economía informal ganan menos que el promedio de ingreso nacional".

En América Latina y el Caribe se experimentaron transformaciones profundas en los ámbitos social, político y económico en los últimos decenios. Pollack y Jusidman (1997) plantean que se agudizó el empobrecimiento de la región a la vez que se produjo una incorporación masiva de las mujeres al mercado de trabajo, lo que ha traído consigo una feminización del sector informal. Para mejorar la calidad de vida de la población es imprescindible mejorar la situación de su población femenina, y a ese fin, es importante perfeccionar la inserción laboral, para lo cual 
se requiere contar con diagnósticos acabados que consideren sus especificaciones y las barreras que enfrentan para acceder a empleos que permitan escapar de la situación de pobreza.

De la misma manera, autoras como Chant y Pedwell (2008) argumentan que, dada la diversidad de la economía informal, las mujeres se encuentran en desventaja con respecto a los hombres, este análisis se remite al tipo de actividades informales que realizan las mujeres, como el trabajo de servicio doméstico, el trabajo independiente y el trabajo no remunerado en pequeñas y medianas empresas de ámbito doméstico. Por su parte la Organización Internacional del Trabajo (2002, p. 24) considera que:

El empleo informal puede tener características como falta de protección en casos como el no pago de salarios, obligación de hacer sobretiempo o turnos extraordinarios, despidos sin aviso ni compensación, condiciones de trabajo inseguras y ausencia de beneficios como las pensiones, el reposo por enfermedad o el seguro de salud. Las mujeres, los migrantes y otros grupos de trabajadores vulnerables que no tienen otras oportunidades se ven obligados a aceptar trabajos en condiciones de informalidad.

Esta investigación tiene como objetivo mostrar el estado del arte de los conceptos de economía informal o subterránea, trabajo de mujeres y criticas al concepto de informalidad. Por último, se realiza un análisis de la economía informal y trabajo informal de mujeres desde los aportes teóricos expuestos en este estudio.

\section{TRABAJO DE MUJERES}

Ester Boserup, en 1970, centra la atención sobre la participación de las mujeres en las actividades económicas. Boserup (como se cita en Benería y Sen, 1982) identifica la división del trabajo por sexo y edad de formas masculinas y femeninas en la agricultura; los efectos negativos del colonialismo sobre la condición de las mujeres; la ausencia de reconocimiento del papel de las mujeres en las cifras sobre actividad económica y la relación de patrones de trabajo agrícola con otros patrones demográficos.

Boserup (1970) encontró que, desde la época de la colonia, las costumbres europeas perturbaron las organizaciones y arreglos originales en los que la mujer tenía un papel más relevante. Se refiere al deterioro progresivo del estatus de la mujer - pérdida de poder, independencia y propiedad-como consecuencia de la introducción de patrones europeos, y por su transformación de cultivadoras autónomas a ayudantes familiares sin asistencia técnica. 
Barquet (citado por García, 2015, p. 14) sostiene que, desde los inicios de la humanidad "la persistencia de patrones socioculturales de género que se asignan a una mujer son las funciones de cuidadora/nutricia, centradas en su función biológica reproductiva. Por lo tanto, hace que las mujeres carguen con la responsabilidad del mantenimiento, reproducción y reposición de la fuerza de trabajo, que se exacerban en condiciones de crisis".

El Grupo Interdisciplinario sobre Mujer, Trabajo y Pobreza (Gimtrap, 1999) analiza las políticas hacia mujeres durante los años setenta, ochenta, noventa y mitad del siglo veinte. En los años setenta las políticas hacia las mujeres parecían comprendidas entre las políticas sociales o eran programas de asistencia vinculados con las instituciones de salud y de protección de familia. Desde esa misma época, se generalizó la perspectiva de incorporación de las mujeres al desarrollo, pero la inclusión del tema en los planes y programas de largo alcance se diluyó dentro de la planificación global. Para los años ochenta, los problemas de pobreza creciente estimularon el diseño de programas enfocados en las mujeres pobres, con objetivos asistenciales a partir del rescate del concepto de políticas públicas, de la incorporación en ellas del enfoque de género y de las proporciones que privilegian la equidad social.

Durante la segunda mitad del siglo veinte, el proceso de industrialización, urbanización y el derecho al sufragio, estimularon la expansión de la actividad económica femenina y, en ese marco, se iniciaron movimientos por una mayor igualdad de empleo y servicios entre hombres y mujeres, que culminaron con la Conferencia Mundial del Año Internacional de la mujer en $1975^{1}$.

Autores como Benería y Roldan (1992) argumentan que, tras varias décadas de políticas que han intentado incorporar a las mujeres al proceso de desarrollo (Mujeres en Desarrollo, MED; Woman in Devolopment, WID; Women And Development, WAD), los resultados no mostraron haber cambiado mucho en el Tercer Mundo, por el contrario, han tenido repercusiones distintas en hombres y en mujeres, y a menudo el efecto ha sido negativo para las mujeres.

Las perspectivas de incorporación a las cuales se referían los autores, hablan del reconocimiento de la exclusión de las mujeres de los beneficios de la modernización, solucionando dicha premisa desde la educación, la inserción laboral y la promoción de cambios legislativos a favor de las mujeres, o mediante un análisis del desarrollo del país, sin tener presente la subordinación de género que transciende lo económico.

Las Naciones Unidas han organizado cuatro conferencias mundiales sobre la mujer, que se celebraron en Ciudad de México (1975). 
Al respecto Young (1991) y Moser (1989), coinciden en señalar la necesidad de distinguir entre las condiciones de las mujeres, de la posición de la mujer que daría cuenta de su situación relacional comparativa con la de los hombres y su posición de género. Young (1991) argumenta que esta distinción cobra importancia para el análisis de las políticas de desarrollo para las mujeres, a partir de la atención de las necesidades prácticas cotidianas.

Barquet (1991) expone que no es posible hablar de mujer como unidad conceptual, sin incluir elementos tales como clase, raza, estado civil, edad y posición en el ciclo vital. La literatura feminista señala la subordinación y opresión como experiencia de las mujeres, aunque las formas concretas que asume la subordinación varíen en distintas culturas y épocas históricas. Así las cosas, se identifica, por un lado, la división sexual del trabajo que asigna a las mujeres el grupo de las tareas domésticas de reproducir y mantener las generaciones, excluyéndolas del disfrute del valor y del ejercicio del poder y, por otra parte, la organización de la sexualidad y la procreación que permite la apropiación individual de las capacidades generativas, lo cual dificulta la autonomía y la libertad de las mujeres. Parella (2000, pp. 280-281) argumenta que:

Las mujeres son discriminadas en el mercado de trabajo de las sociedades occidentales con base en la asunción patriarcal que considera que el rol natural de la mujer está en la esfera reproductiva, por lo que va a ser menos productiva que un hombre en determinados trabajos remunerados y, además, su actividad se va a ver afectada por sus responsabilidades familiares, en términos de movilidad, estabilidad y eficiencia. La concentración primordial de la mujer en el área de la reproducción la convierte en trabajadora secundaria o ausente en el área de la producción. Este proceso de segregación horizontal es el resultado de la existencia de actividades consideradas «femeninas» en el imaginario social, en las que las mujeres prolongan sus habilidades como madres, esposas y cuidadoras, adquiridas a través de la socialización diferencial de género. Las mujeres trabajadoras se sitúan así en los estratos más bajos de la estructura ocupacional, en aquellas actividades más precarizadas, menos remuneradas y menos valoradas socialmente.

Rosenhouse (1988) propuso que la jefatura no se midiera por la mayor generación de ingresos, sino por los esfuerzos y el compromiso con el bienestar del hogar, entendiendo por esfuerzo, el número de horas destinadas en el mercado laboral, lo que además permite corregir los sesgos de medición de ingreso para los trabajadores independientes. El concepto de jefatura analizado en el estudio de Rico et al. (1999) supone también la presencia de relaciones de poder y jerarquía, designando como jefe a la persona de mayor autoridad y capacidad económica, lo cual excluye la presencia de relaciones intergenéricas e intergeneracionales menos jerarquizadas 
y jefaturas compartidas entre cónyuges padres e hijos u otros arreglos. En el sector informal, en especial en las actividades de comercio, no existe evidencia para afirmar que el pago por producto dependa del sexo del vendedor sino de las condiciones de oferta y demanda del producto.

\subsection{Ocupaciones de las mujeres}

En este aspecto se han revisado trabajos de Htun (2003), Bustos (1994) y Arango (2011), que son los trabajos más citados en estudios de ocupaciones de mujeres. Htun en su artículo Las mujeres y la política, argumenta que la participación de la mujer en las políticas democráticas en América Latina es motivo de celebración y de preocupación. Su mayor intervención en la toma de decisiones, los compromisos explícitos sobre equidad de género adquiridos por los gobiernos y la atención oficial a problemas de género como la violencia familiar y sexual, son motivos de celebración. La situación de muchas mujeres en América Latina ha mejorado desde algunas décadas. Sin embargo, han sido mediocres ya que los derechos de la mujer se han convertido en algo más abstracto que concreto. Htun argumenta que lo que se requiere es una transformación práctica social alrededor de las relaciones de género provocadas por los cambios demográficos, socioeconómicos y tecnológicos que pueden filtrarse para influir en la voluntad de los hombres y mujeres para someterse a la autoridad de las mujeres.

La socióloga y feminista Luz Gabriel Arango en su artículo titulado A la sombra de los padres fundadores de la sociología, publicado en el libro El género: una categoría útil para las ciencias sociales, utiliza la categoría de género para mostrar el carácter androcéntrico de la sociología desde dos perspectivas: la construcción del canon y la formación de la identidad profesional en los procesos de formación universitaria. De la misma manera, en la vertiente hegemónica del pensamiento sociológico en América Latina y en Colombia, la producción de las mujeres y la crítica feminista han estado presentes con escaso reconocimiento. Por este motivo, la autora incorpora la producción sociológica de las mujeres, tanto de aquellas que fueron excluidas de la historia oficial de la sociología dominante como de las autoras latinoamericanas y colombianas.

Tanto el campo político como el campo científico se constituyeron históricamente bajo la hegemonía masculina. Para Arango (2011) Las mujeres lograron con dificultad acceder a esos campos, y una vez dentro, en proporciones numéricas minoritarias, se convirtieron en recién llegadas en búsqueda de reconocimiento de su legitimidad y autoridad. Conceptos psicológicos como el de Stoller (1985) sobre la identidad personal y la diferenciación entre la biología y la cultura, resultan 
interesantes de considerarse para el estudio. Al igual que los de Simone de Beauvoir quien denomina a la mujer como "una identidad" y no como una identidad natural y un proyecto, por lo tanto está en desventaja social con respecto al hombre. Por su parte Irigaray (1992) concuerda con el planteamiento de Simone de Beauvoir, en lo siguiente:

Las mujeres tienen ocupaciones heteroasignadas en la vida cotidiana, por lo tanto, las mujeres tienen que trabajar en ocupaciones de servicio a favor del mantenimiento de la paz, la salud, el ambiente saludable, los bienes acordes con las necesidades de la vida humana, animal y vegetal o las opciones humanitarias en contraste con las ocupaciones de los hombres. (p. 119).

Se trata de una realidad que muestra cómo los espacios, los tiempos y las actividades que desarrollan las mujeres para satisfacer las necesidades del núcleo familiar, y para mantener su unidad, no son compartidos por los varones; y de una realidad llena de actividades en las que se hace indispensable la presencia e iniciativa de las mujeres, aunque sus alcances sociales y económicos no sean reconocidos como ocupaciones. Bustos y Palacio (1994) en su libro El trabajo femenino en América Latina: los debates en la década de los noventa muestran la posición de la mujer dentro del binomio capital- trabajo, en el marco de la reconversión productiva y del proceso de globalización del capital. Los autores abordan la discusión sobre los nuevos paradigmas productivos y su relación con el empleo femenino, sostienen que el mundo laboral de la mujer latina es muy amplio, ya que se puede encontrar en todos los sectores productivos, servicios, manufactura, construcción, comunicaciones, casi en cualquier puesto de trabajo. Sin embargo, son pocas las posibilidades de desarrollo y crecimiento profesional que la mujer tiene en América Latina, ya que su trabajo e ingreso se ven como complemento al del proveedor principal de la familia.

\section{CONCEPTO DE INFORMALIDAD}

Economía informal o subterránea es el nombre que se le da a un gran número de actividades que están catalogadas dentro del sector informal de la economía. Estos tipos de economías carecen de reglamentación institucional, de tecnologías, de formas avanzadas de producción y de una división del trabajo establecida, según World Trade Organization (WTO, por sus siglas en inglés) (2009).

Williams (2015, p. 7) entiende por sector informal el sector privado no incorporado, empresas que no están registradas, al igual que pequeñas en términos del número de personas empleadas. El empleo informal, por su parte, se refiere a trabajos que carecen de protecciones sociales o legales básicas o beneficios de empleo y se 
encuentran en el sector formal, el sector informal o los hogares.

La tabla 1 explica dicho concepto. La empresa es la unidad de análisis, el sector informal abarca el empleo informal y formal en las empresas del sector informal $(\mathrm{A}+\mathrm{B})$; por su parte el empleo informal abarca los empleos informales tanto en empresas informales como formales (A + C) (Williams, 2015, p. 7).

Tabla 1. La anatomía de la informalidad.

\begin{tabular}{|l|c|c|}
\hline \multicolumn{1}{|c|}{ Unidades económicas } & Empleos informales & Empleos formales \\
\hline Unidades económicas informales & $\mathrm{A}$ & $\mathrm{B}$ \\
\hline Unidades económicas formales & $\mathrm{C}$ & $\mathrm{D}$ \\
\hline
\end{tabular}

Fuente: Organización Internacional del Trabajo, 2012 como se cita en Williams $(2015$, p. 7).

Así mismo, la OIT (citada por Rodríguez y Calderón, 2015, p. 42) estableció que:

Se considera que los asalariados tienen un empleo informal si su relación de trabajo, de derecho o, de hecho, no está sujeta a la legislación laboral nacional, al impuesto sobre la renta, a la protección social o a determinadas prestaciones relacionadas con el empleo (preaviso al despido, indemnización por despido, vacaciones anuales pagadas o licencia pagada por enfermedad, etc.).

Las razones pueden ser las siguientes: la no declaración de los empleos o de los asalariados; empleos ocasionales o de limitada duración; empleos con un horario o un salario inferior a un límite especificado (por ejemplo para cotizar a la seguridad social); el empleador es una empresa no constituida en sociedad o una persona miembro de un hogar; el lugar de trabajo del asalariado se encuentra fuera de los locales de la empresa del empleador (por ejemplo, los trabajadores fuera del establecimiento y sin contratos de trabajo); o empleos a los que el reglamento laboral no se aplica, no se hace cumplir o no se hace respetar por otro motivo. Los criterios operativos para definir empleos asalariados informales deberían determinarse en función de las circunstancias nacionales y de la disponibilidad de información.

Para el caso de Colombia, el DANE estableció que el empleo informal engloba las características de los empleos de los trabajadores. Así, para el DANE (2009) las características que permiten precisar la definición de empleo informal se describen a continuación: los empleados particulares y los obreros que laboran en establecimientos, negocios o empresas que ocupen hasta cinco personas en todas sus agencias y sucursales y los trabajadores familiares sin remuneración. Al querer medir la informalidad Mejía y Posada (2007) argumentan que el grado de informalidad se suele medir como la proporción de la producción del trabajo informal con respecto al total. Por lo tanto, se debe en gran medida, al cambio de política económica en los últimos 15 años en el país, y a que la informalidad está asociada a la facilidad 
de evasión de normas propias de la producción en pequeña escala. La informalidad puede ser un refugio involuntario. Se fija un salario excesivo, lo que genera el aumento de la oferta. Y, en el caso de la informalidad como una estrategia voluntaria, mercados flexibles, completos e informados.

Hart (1970) analiza los negocios de pequeña escala en Ghana y encuentra que había una fracción sustancial de miembros de la fuerza laboral urbana que trabajaban por fuera del mercado laboral en negocios de pequeña escala y por cuenta propia. La Organización Internacional del Trabajo (1972) para el caso de Kenia consideró informal toda actividad que evade impuestos y no se somete a regulación estatal. La informalidad es sinónimo de pobreza es decir de una válvula de escape ante la perspectiva de desempleo. Marginalidad y exclusión. Tokman (1982) mencionó que el sector informal está caracterizado por bajos niveles de productividad y por la incapacidad de acumular capital físico y humano a gran escala. (Mejía y Posada, 2007, p. 5).

Hirschman (1970) realiza un análisis diferente al de la OIT. Para este autor la evasión de impuestos es más viable para los empresarios de las pequeñas y medianas empresas y por tanto la informalidad se escoge como una forma de realizar su trabajo lejos de la formalidad, todo lo anterior debido a la incapacidad del Estado para cobrar el fisco a este tipo empresas. Mincer (1976), realizó una investigación mediante el uso de un modelo de dos sectores para establecer un salario mínimo, utilizó un sector protegido y otro no protegido, el protegido cumplía la norma y el no protegido no, de esta manera analizaron los efectos entre lo formal y lo informal.

De Soto (1989), perteneciente a la escuela legalista e iniciador del análisis sobre la informalidad en países en desarrollo, incluye en su énfasis las comparaciones individuales entre beneficios y costos de evadir las normatividades y las relaciones entre informalidad y desarrollo económico. Es decir, el estar o no en el mercado informal se debe a una decisión que busca evitar la carga tributaria. Rauch (1991) realizó su análisis del mercado laboral entre lo formal y lo informal. Este argumenta la teoría de búsqueda de trabajos y trabajadores y del mercado segmentado, encontrando que, precisamente, los trabajadores optan por trabajos informales por facilidad, este autor es seguidor de De Soto.

Loayza (1996), a partir de una muestra de países latinoamericanos, explicó la informalidad y sus efectos usando la premisa de la evasión de impuestos. Su estudio concluye con la afirmación que la informalidad frena el crecimiento económico. Maloney (1998) y (1999) discrepa de Loayza en la reducción del tamaño del sector informal. El autor asegura que esto generaría un alto costo social, en consecuencia, afirma que es adecuado para las economías permitir cierto grado de informalidad. Por su parte, Perry et al. (2007) analizan la informalidad como un enfoque individual 
y lo denominan como un refugio involuntario, debido a la necesidad de incumplir la normatividad. Aunque es importante mencionar que este trabajo no tiene en cuenta interrelaciones de informalidad empresarial.

Castells (1989) planea la economía informal como un proceso generador de ingresos caracterizado por eludir las regulaciones de las instituciones de la sociedad. En esta misma corriente se encuentra De Soto (1989), quien considera que la informalidad son todas aquellas actividades que desobedecen las regulaciones estatales. La diferencia en estar o no se encuentra en el estatus formal. (Mejía y Posada, 2007, p. 5).

Sethuraman (1981) y Bromley (1978) cuestionan el sector informal, lo denominan como: "un universo amplio de actividades por lo que las definiciones dadas hasta podrían correr el riesgo de ser simplistas y omitir variables importantes". Autores como Robbins y Ruiz (2007) realizan un análisis endógeno de la informalidad. Hablan del enforcement denominado como un "elemento de la informalidad donde la probabilidad de que un evasor de una norma fiscal sea detectado". A través de una distribución aleatoria determinan que la probabilidad de que un evasor de tributo sea detectado se debe a la distribución exógena entre los recursos destinados al pago de tributo y las empresas formales e informales. Así mismo, Antunes y Cavalcanti (2007) realizan un análisis de la informalidad desde el equilibrio general. Para ellos el fenómeno de la informalidad es un factor exógeno de acuerdo al grado de enforcement en las contrataciones formales. Mejía y Posada (2007) estudian los determinantes del grado óptimo de enforcement de las normas y regulaciones cuya violación define a las empresas informales. En consecuencia, el gobierno escoge un grado de enforcement y por esta vía acepta un nivel óptimo de informalidad. Cabe destacar que Mejía y Posada (2007), no concuerdan con Becker (1968) cuando se hace el análisis del nivel óptimo de enforcement de la ley penal. Para ellos, el enforcement no es de tipo macroeconómico, sino micropenal, al querer cuantificar la frecuencia de los crímenes y las violaciones de ley.

Con respecto a los estudios de Colombia, investigadores como Cárdenas y Mejía (2007, p. 7), enuncian que el tamaño de la economía informal en Colombia en el periodo de 1989-1993 fue $35 \%$, porcentaje inferior al promedio latinoamericano, que fue del $39 \%$. De acuerdo con Schneider (2003), en cambio en 2002-2003 creció de manera significativa. Esto se debe a que se encuentran estimaciones de informalidad en diversos países desarrollados y en desarrollo. Antunes (2014) en su artículo La nueva morfología del trabajo y sus principales tendencias, informalidad, inforproletariado, inmaterialidad y valor argumenta que la economía del empleo es algo presente en la propia lógica del sistema de metabolismo social del capital. Por su parte Mészáros (1995, p. 61), expone que "la reducción del trabajo vivo no significa pérdida de centralidad del trabajo abstracto en la creación del valor, que hace mucho dejó de ser resultado de una agregación 
individual de trabajo, para convertirse en trabajo social, complejo combinado y que con el avance tecno-informacional-digital, no deja de ser más complejo y potenciarse".

De la misma manera, Antunes (2014, p. 205) argumenta que:

Hay diferentes formas de informalidad entre las cuales se encuentran, los trabajadores informales tradicionales insertos en actividades que requieren baja capitalización y orientadas a la obtención de una renta para consumo individual y familiar. Viven de su fuerza de trabajo y sirven de auxilio en el trabajo familiar o de ayudantes temporales. En este universo están varios tipos de trabajadores, los primeros son los trabajadores menos inestables estos poseen un mínimo conocimiento profesional y los de medios de trabajo. En la mayoría de los casos, desarrollan sus actividades en el sector de los servicios. Es el caso de las costureras, los albañiles, los jardineros, los vendedores ambulantes de artículos de consumo más inmediato (alimentos, vestuario, calzado) y de artículos de consumo personal, vendedores de la calle, empleados domésticos y los talleres de reparación.

Para culminar se presenta la definición de informalidad de la Organización Internacional del Trabajo (2013, p. 14):

\begin{abstract}
El sector informal puede describirse en términos generales como un conjunto de unidades dedicadas a la producción de bienes o a la prestación de servicios con la finalidad primordial de crear empleos y generar ingresos para las personas que participan en esa actividad. Estas unidades funcionan en pequeña escala, con una organización rudimentaria, en la que hay muy poca o ninguna distinción entre el trabajo y el capital como factores de producción. Las relaciones de empleo -en los casos en que existan- se basan más bien en el empleo ocasional, el parentesco o las relaciones personales y sociales, y no en acuerdos contractuales que supongan garantías formales.
\end{abstract}

\title{
3. CRÍTICAS AL CONCEPTO DE INFORMALIDAD
}

Para autores como Carlos Salas, los informales son los individuos que buscan obtener un ingreso por vías legales y que terminan haciendo cualquier actividad para sobrevivir, es decir:

Los trabajadores domésticos remunerados; los trabajadores por cuenta propia o trabajadores familiares no remunerados; los trabajadores asalariados que carecen de condiciones adecuadas de trabajo, sea en términos de salario, contrato laboral o pago de prestaciones; los trabajadores que realizan alguna tarea en el marco de la subcontratación con empresas capitalistas; las microempresas; todos los establecimientos que no cumplen con alguna disposición legal relativa al ámbito laboral; y aquellas unidades que incumplen alguna regulación gubernamental, por ejemplo, el registro fiscal. (Salas, 1992) citado por Campos, (2008, p.4) 
De este modo, es frecuente encontrar que hay determinada población que no es incluida como informal, lo cual no implica dejar a un lado la noción de informalidad por su anarquía conceptual (Salas, 2006), la informalidad no es una categoría de análisis estática, monolítica, unívoca, ni sobre la cual exista consenso (Sánchez, 2013, p.39). En consecuencia, Salas se opuso al uso del término de informalidad e hizo la propuesta de reemplazarlo por el título más genérico de "estudio de las microunidades" (Salas, 1992) citado por Campos (2008, p.4).

La noción de informalidad es desde el punto de vista conceptual algo muy débil, que, si bien sirvió para llamar la atención de ciertos fenómenos, ha traído más confusión que luz al estudio de problemas ocupacionales, los cuales plantean la imperiosa necesidad de abandonar el concepto. (Salas, 2006). Este mismo autor continúa su crítica al concepto de informalidad categorizándolo en tres grandes grupos:

1. La presencia de criterios múltiples, cada uno de los cuales puede cumplirse de manera independiente de los demás.

2. La imposibilidad de separar al universo de referencia en dos sectores ajenos y complementarios.

3. La imprecisión en el universo de referencia. Ya que se utiliza el establecimiento, el hogar o el individuo. (Salas, 2006, p.15).

Existen otros críticos al concepto de informalidad, Haan en 1989 se refiere a una idea de informalidad que descansa en la existencia de dos sectores complementarios de la economía, este afirma: "en los más de quince años que han trascurrido desde que se introdujo el concepto de sector informal, ha sido imposible traducir la noción anterior en una definición aceptable, consistente y utilizable del empleo informal" (Haan, 1989, p. 6). Por su parte Sethuraman (1976, p. 80) acepta la existencia de una dicotomía que separa las actividades económicas entre formales e informales. La dicotomía significa que la noción de informalidad es dualista. La noción de informalidad se ubica en el centro de la formulación de políticas de desarrollo, ya que permite identificar a los destinatarios de los programas de desarrollo y la caracterización numérica del sector, los cuales fueron un punto de partida necesario en el intento de formular políticas eficaces (Salas, 2006, p.20).

Al tomar como referencia, la anterior afirmación de Sethuraman en 1976 y posterior análisis de Salas en 2006, se puede argumentar que, con una adecuada caracterización del sector en términos numéricos, es de uso primordial, el formular políticas públicas que busquen mitigar la informalidad en las mujeres informales de Colombia. 
Sánchez (2013) en su artículo "Enfoques, conceptos y metodologías de medición de la informalidad laboral en Colombia", muestra diferentes perspectivas de análisis sobre informalidad laboral. El autor enfatiza en las tensiones entre los diferentes enfoques y las diferencias en la mensurabilidad y encuentra que la informalidad en Colombia puede ser desde el 16\% hasta el 59\%. Además, encuentra que:

Las tasas de informalidad cercanas no incluyen a los mismos individuos, lo que critica bajo el argumento de generar una "dificultad colosal" a la hora de utilizar los estudios de informalidad para la generación de políticas públicas. Para el autor la informalidad laboral se presenta en situaciones heterogéneas: para unas perspectivas es característica de las escalas de producción, para otras de la existencia de afiliación y/o contribución al sistema de seguridad social, y otras lo conciben como una situación propia de determinadas actividades, del tipo de relación laboral o de las relaciones con el Estado y las instituciones. Sánchez (2013, p.38)

Según Sánchez las diferentes aplicabilidades de la informalidad en los estudios sobre empleo en Colombia tienen dos problemas: "el primero es concebir la informalidad laboral como una problemática unidimensional y vinculada a fenómenos económicos, por lo que, para la mayoría de estudios, los trabajadores informales no son más que un dato que hay para analizar y problematizar, y dejan a un lado aproximaciones de otras ciencias sociales, que por cierto en Colombia son muy reducidas". Sánchez (2013, p 39)

El segundo problema que plantea Sánchez (2013) es que la mayoría de estudios sobre informalidad laboral buscan homogeneizar algo heterogéneo por antonomasia.

Estudios como los de Flórez (2002) y Uribe et al., (2006) tratan de categorizar la informalidad, dejan de ser categorías generales que poco se centran en las características reales de los trabajadores y la forma en que se desenvuelven en el mercado laboral. Es importante resaltar que Sánchez (2013) argumenta que, a pesar de las diferencias de enfoque conceptual y metodológico, se puede ubicar un perfil mayoritario de los trabajadores informales, a saber: -Concentración en edades adultas -Niveles educativos medios y bajos -Actividades comerciales - Trabajadores con niveles de ingreso bajo. (Sánchez, 2013, p. 39).

Sánchez (2013) asegura que existen otros rasgos de los trabajadores y sus ocupaciones en los que, a partir de la orientación operativa, cambia su influencia sobre la probabilidad de informalidad, tal es el caso de la posición en el hogar. De la misma manera, argumenta que la probabilidad de tener trabajos informales es mayor para las mujeres que para los hombres. 


\section{ANÁLISIS DE LA ECONOMÍA INFORMAL Y TRABAJO INFORMAL DE MUJERES}

Según Christopher Pissarides, premio Nobel de economía del año 2010, "la gente se vuelve informal para evadir impuestos; al empleo le ponen muchos tributos, lo cual no ayuda a frenar la informalidad" (El Espectador, 2013). Al analizar la convergencia entre economía informal y trabajo informal de mujeres, es importante evidenciar como se presentó este fenómeno en Colombia entre los años 2001 y 2017. Según cifras del DANE (2009), la informalidad tuvo una tendencia constante entre los años 2001 al 2003. Durante los años 2004 al 2008 se presentó una disminución importante hasta alcanzar una tasa de 50,9\%. No obstante, a finales del último periodo presidencial de Álvaro Uribe Vélez, la informalidad subió hasta alcanzar una tasa del 68,5 \% en el año 2010. En el primer periodo presidencial de Juan Manuel Santos, la informalidad en Colombia presentó una tendencia por encima de los cincuenta puntos porcentuales a excepción del año 2014, cuando alcanzó una cifra del 49,4 \%, tendencia que siguió a la baja hasta llegar en el año 2017 al 48,4\%. Ver gráfica 1.

\section{Gráfica 1. Tasa de informalidad en Colombia.}

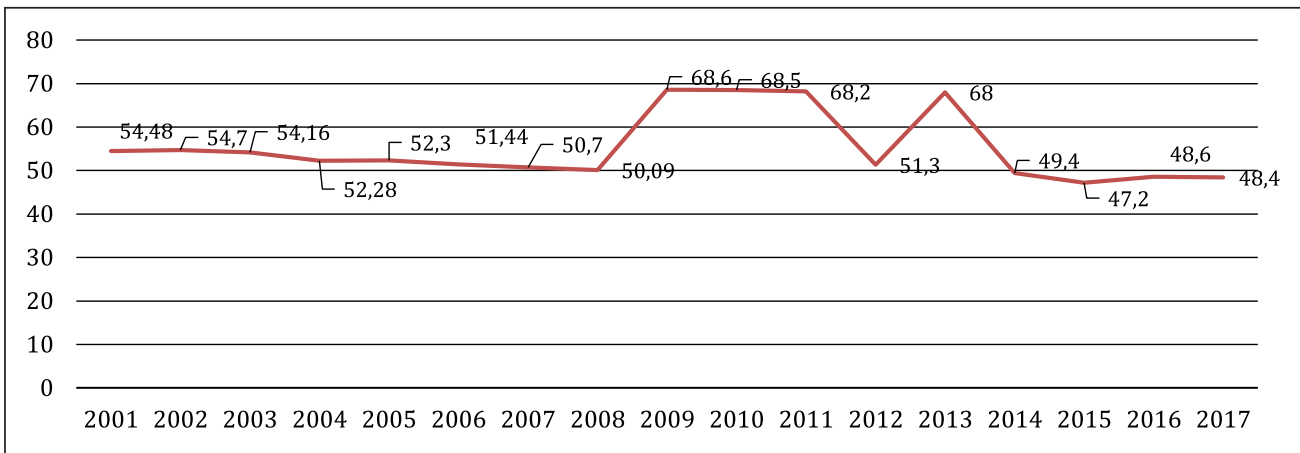

Fuente: elaboración propia con base en DANE (2017).

Según datos del DANE (2017), el número de mujeres que participan en el mercado laboral informal del país presentó un alza desde el año 2007 al 2017. Para el caso del año 2016 al 2017, el número de mujeres informales en el país subió en 13.757 mujeres. Esta tendencia al alza permite argumentar que la presencia de las mujeres en la economía informal del país es cada año más alto (gráfica 2).

El crecimiento del número de mujeres que trabajan en el mercado laboral informal en Colombia es consecuente con los diferentes aportes teóricos planteados al inicio de esta investigación, es el caso de Pollack y Jusidman (1997) y su planteamiento sobre la feminización del mercado de trabajo informal, como consecuencia de la incorporación masiva de mujeres al mercado laboral latinoamericano. Para el caso 
de Colombia Rosales (2003, p. 3) correctamente estimaba que "la mayoría de los trabajadores informales serían asalariados (62 \%) y de sexo femenino".

Gráfica 2. Número de mujeres con trabajo informal en Colombia

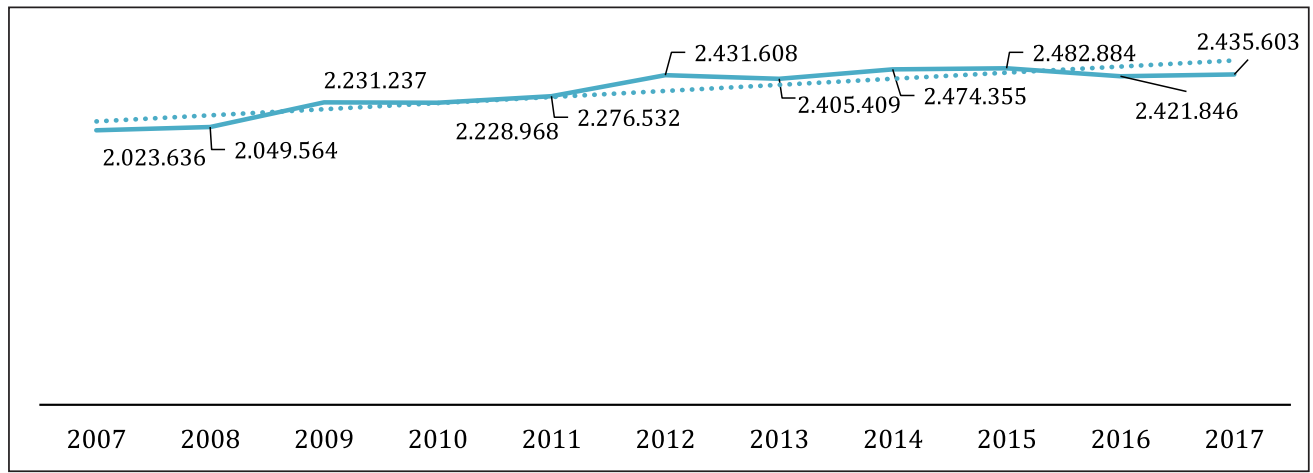

Fuente: elaboración propia con base en DANE (2017).

Esta investigación simpatiza con la posición de la escuela legalista liderada por De Soto (1989), quien argumenta que el fenómeno de la informalidad es consecuencia de la desobediencia social. En este orden de ideas, las mujeres que participan en la informalidad, lo hacen de una manera libre y racional, basadas en sus preferencias personales que buscan la maximización de la utilidad. La presencia en la economía informal se debe en principio a una libre decisión, basada en la evasión de impuestos (ver gráfica 3).

Gráfica 3. Análisis del concepto de economía informal de la escuela legalista y el trabajo informal mujeres.

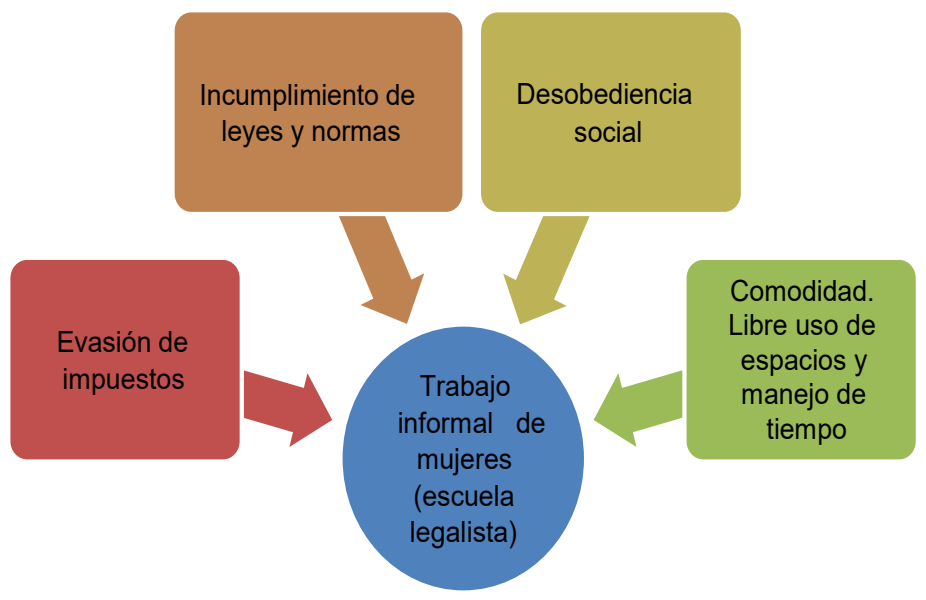

Fuente: elaboración propia con base a De Soto (1989) 
No obstante, esta investigación discrepa con el planteamiento de Bustos y Palacio (1994). Los autores sostienen que "el mundo laboral de la mujer latina es muy amplio, ya que se puede encontrar en todos los sectores productivos, servicios manufactura, construcción, comunicaciones, casi en cualquier puesto de trabajo". Los autores, suponen que las mujeres tienen la posibilidad de acceder a trabajos formales de forma fácil y sin restricciones, pero en la práctica y específicamente en el caso de Colombia las cifras del DANE entre los años 2007 al 2017 evidencian que las mujeres no se están empleando en los sectores formales de la economía, sino por el contrario en el mercado laboral informal, cuya consecuencia, es la intensificación del fenómeno de la informalidad femenina en Colombia y por lo tanto de la pobreza de las mujeres. $\dot{c}$

\section{CONCLUSIONES}

Esta investigación es una recopilación de algunas de las diferentes posturas doctrinales referentes a los conceptos de economía informal, trabajo informal de mujeres y criticas al concepto de informalidad. La elección de estas posturas fue el resultado de un ejercicio de revisión bibliográfica de los aportes más citados en revistas especializadas y libros que son referentes actuales de dichos conceptos.

Al analizar las diferentes postulaciones sobre economía informal, se afirman dos cosas. La primera, que algunos autores plantean el fenómeno de la informalidad como un refugio involuntario donde las personas con pocas o ninguna opción en el mercado formal de trabajo ingresan para sobrevivir, lo que es parte de una válvula de escape a su destino, así lo señalan autores como Mejía y Posada (2007), Hart (1970), Tokman (1982) y Perry y otros (2007).

Por otra parte, existe la postura de la escuela legalista cuyo mentor es De Soto (1989), que parte de la premisa de que la informalidad contempla todas aquellas actividades fuera de las regulaciones estatales denominadas como leyes y normas, con el fin de evitar el pago de impuestos. Es decir, las mujeres deciden hacer parte del sector informal de la economía a partir de una libre elección, basándose en sus situaciones individuales y manteniendo el concepto de las distancias sociales, la conformidad y la comodidad. Buscando principalmente, evitar el pago de impuestos, lugares y horarios fijos.

De la misma manera, este estudio concuerda con la premisa de Parella (2000), quien afirma que las mujeres trabajadoras se sitúan en los estratos más bajos de la estructura ocupacional, en aquellas actividades más precarizadas, menos remuneradas y menos valoradas socialmente. Así como lo supone Barquet (1991), 
las tareas del hogar son oficios derivados de la creencia de que muchas actividades se atribuyen naturales a las mujeres y por lo tanto genera que estas decidan realizar labores informales. Labores sin prestaciones de ley, ni seguridad ocupacional, en su mayoría sin requerir el cumplimiento de horarios ni lugares fijos, lo que imposibilita la tributación y por lo tanto la formalización de labores.

En futuras investigaciones se pueden realizar análisis relacionados con la convergencia en la participación de las mujeres en el mercado laboral informal y las preferencias sociales a partir de un estudio cuantitativo. Dichos aportes serían de gran utilidad en la construcción de políticas públicas que permitan que las mujeres ingresen al mercado laboral formal. Por otra parte, se sugiere realizar investigaciones donde se conecte esta temática con otras áreas del conocimiento, tales como estudios de género, psicología, sociología y economía del comportamiento. Con el fin de evaluar las hipótesis de los autores mencionados en esta investigación. Todo lo anterior, con el fin de poder aproximarse a las verdaderas razones de la existencia de la economía informal en países latinoamericanos y especialmente a la participación mayoritaria de las mujeres.

\section{BIBLIOGRAFÍA}

Antunes, António y Cavalcanti, Tiago (2007). Start Up Costs, Limited Enforcement, and the Hidden Economy. En: European Economic Review, vol. 51, n. ${ }^{\circ}$ 1, p. 203-224.

Arango, Luz y Viveros Mara (2011). El género: una categoría útil para las ciencias sociales. Bogotá, Colombia: Universidad Nacional, 372 p.

Barbieri, Teresita (1982). Derechos humanos de las mujeres y políticas de población: una relación compleja. Consejo Nacional de Ciencia y Tecnología: Ciudad de México, México: Il Reunión de investigación demográfica en México, p. 785-788.

Barquet, Mercedes (1991). La condición de la mujer en México: una vista panorámica. Taller sobre análisis de género. México D.F, México: Mexfam, 158 p.

Barquet, Mercedes (1994). Condicionantes de género sobre la pobreza de las mujeres. En: Alatorre, Javier; Careaga, Gloria; Jusidman, Clara; Salles, Vania.;Talamantes, Cecilia y Townsend, John (Coords.). Las mujeres en la pobreza. México D.F, México: El Colegio de México, p. $73-90$.

Becker, Gary (1968). Crime and Punishment: An Economic Approach. En: The Journal of Political Economy, vol. 76, n. 2, marzo-abril, p.169-217.

Benería, Lourdes y Roldán, Martha (1992). Las encrucijadas de clase y género. Trabajo a domicilio, subcontratación y dinámica de la unidad doméstica en la ciudad de México. México D.F, México: Fondo de Cultura Económica, 224 p.

Benería, Lourdes y Sen, Gita (1982). Acumulación, reproducción y el papel de la mujer en el desarrollo económico: una revisión a Boserup. En: Magdalena León (ed.). Las Trabajadoras del agro (vol. II). Bogotá, Colombia: ACER, p. 23-38. 
Revisión de la literatura sobre economía informal y trabajo de mujeres

Boserup, Ester (1970). Women's Role in Economic Development. Londres, Reino Unido: George Allen and Unwin Ltd, 283 p.

Bromley, Ray (1978). Introduction: The Urban Informal Sector: Why is it worth Discussing? En: World Development, vol. 6, n. ${ }^{\circ}$ 9-10, p.1033-1039.

Bustos, Beatriz y Palacio, Germán (1994). El trabajo femenino en América Latina: los debates en la década de los noventa. Universidad de Guadalajara. Guadalajara, México: Instituto Latinoamericano de Servicios Legales Alternativos, 303 p.

Campos, Guillermo (2008). La economía informal y sus posibilidades de desarrollo. XI Jornadas de Economía Crítica. Universidad Autónoma de Puebla, México, 8 p.

Cárdenas, Mauricio y Mejía, Carolina (2007). La informalidad en Colombia: nueva evidencia Fedesarrollo, Documentos de trabajo marzo, n. ${ }^{\circ} 35$, p. 2-43.

Castells, Manuel; Portes, Alejandro y Benton, Lauren (1989). The Informal Economy: Studies in Advanced and Less Developed Countries. Baltimore, Estados Unidos de América: Johns Hopkins University Press, 327 p.

Chant, Silvya y Pedwell, Carolyn (2008). Las mujeres, el género y la economía informal: evaluación de estudios de la OIT y orientaciones sobre trabajo futuro. Ginebra, Suiza: Oficina Internacional del Trabajo, $68 \mathrm{p}$.

DANE $\square$ Departamento Nacional de Estadística $\square$ (2009). Metodología informalidad gran encuesta integrada de hogares. Bogotá, Colombia: DANE, n. ${ }^{\circ} 83,100$ p.

DANE $\square$ Departamento Nacional de Estadística! (2017). Empleo informal y seguridad social. Bogotá, Colombia: DANE, 12 p.

De Soto, Hernando (1989). The Other Path: The Invisible Revolution in the Third World. Nueva York, Estados Unidos de América: Basic Books, 286 p.

El Espectador (2013). La gente se vuelve informal para evadir impuestos. Bogotá, Colombia, 1 p.

Gimtrap $\square$ Grupo Interdisciplinario sobre Mujer, Trabajo y Pobreza】 (1999). Las mujeres en la pobreza. En: Estudios Sociológicos, vol. XII, n. ${ }^{\circ}$ 36, p. 657-681.

Haan, Hans (1989). Urban informal sector information: Needs and methods. Ginebra, Suiza: Organización Internacional del Trabajo, 44 p.

Hart, Keith (1970). Small scale entrepreneurs in Ghana and Development planning. En: Journal of Development Studies, vol. 6, n. ${ }^{\circ}$ 4, p. 104-120.

Hirschman, Albert (1970). Exit, Voice, and Loyalty: Responses to Decline in Firms, Organizations, and States. Massachusetts, Estados Unidos de América: Harvard University Press, 176 p.

Htun, Mala (2003). Women and Democracy, in Construction Democratic Governance in Latin America. Maryland, Estados Unidos de América: Johns Hopkins University Press, p. 119- 136.

Irigaray, Luce (1992). Yo, tú, nosotras. Madrid, España: Cátedra, 66 p.

Loayza, Norman (1996). The Economics of the Informal Sector: A Simple Model and Some Empirical Evidence from Latin America. En: Carnegie-Rochester Conference Series on Public Policy. vol. 45, p. 129-162. 
Maloney, William (1998). Are Labor Markets in Developing Countries Dualistic? Policy Research Working Paper. Washington, D.C, Estados Unidos de América: Banco Mundial, n. ${ }^{\circ}$ 1941, 39 p.

Maloney, William (1999). Does Informality Imply Segmentation in Urban Labor Markets? Evidence from Sectoral Transitions in Mexico. En: World Bank Economic Review, vol.13, n. ${ }^{\circ}$, p. 275-302.

Mejía, Daniel y Posada, Esteban (2007). Informalidad: teoría e implicaciones de política. En: Borradores de Economía Banco de la República, n. ${ }^{\circ}$ 455, p. 1-33.

Mészáros, Istvan (1995). Beyond Capital: Toward a Theory of Transition. Londres, Reino Unido: The Merlin Press, 994 p.

Mincer, Jacob (1976). Unemployment Effects of Mínimum Wages. En: The Journal of Political Economy. vol. 84, n. ${ }^{\circ}$, parte 2, p. 87-104.

Moser, Caroline (1989). Gender Planning in the Third World: Meeting Practical and Strategic Gender Needs. En: World Development, vol. 17, n. ${ }^{\circ} 11$, p. 1799-1825.

OIT -Organización Internacional del Trabajo- (2013). La medición de la informalidad: manual estadístico sobre el sector informal y el empleo informal. Turín, Italia: Centro Internacional de Formación de la OIT, 374 p.

OIT -Organización Internacional del Trabajo- (2012). Decent work and the informal economy (reporte VI). Ginebra, Suiza: OIT, 133 p.

OIT -Organización Internacional del Trabajo- (2003). Informe de la Conferencia. Decimoséptima Conferencia Internacional de Estadísticos del Trabajo (reporte I). Ginebra, Suiza: OIT, 108 p.

OIT, -Organización Internacional del Trabajo- (2002). El trabajo decente y la economía informal, Conferencia Internacional del Trabajo, 90. ${ }^{a}$ reunión (informe VI). Ginebra, Suiza: OIT, 151 p.

OIT -Organización Internacional del Trabajo- (1972). Employment, income and equality: A strategy for increasing productivity in Kenya. Report of an inter-agency team financed by the United Nations development. Ginebra, Suiza: OIT, 593 p.

Parella, Sonia (2000). El trasvase de desigualdades de clase y etnia entre mujeres: los servicios de proximidad. En: Revista de Sociología, n. ${ }^{\circ}$ 60, p. 275-289.

Perry, Guillermo; Maloney, William; Arias, Omar; Fajnzylber, Pablo; Mason, Andrew y Saavedra-Chanduvi, Jaime (2007). Informality: exit and exclusion (English). Washington, Estados Unidos de América: The World Bank, 270p.

Pissarides, Christopher (2000). Equilibrium unemployment theory. Massachusetts, Estados Unidos de América: MIT Press, 247 p.

Pollack, Molly y Jusidman, Clara (1997). El sector informal urbano desde la perspectiva de género. El caso de México. Santiago de Chile, Chile: Cepal, Naciones Unidas, 71 p.

Rauch, James (1991). Modeling the Informal Sector Formally. En: Journal of Development Economics, vol. $35, \overline{\text { n. }}{ }^{\circ} 1$, p. 33-47.

Rico, Ana; Gómez, Eva; López, Nadia; Castillo, Olga; Alonso, Juan; Galindo, Carolina y Castillo, Sonia (1999). Jefatura, informalidad y supervivencia: mujeres urbanas en Colombia. Bogotá, Colombia: Pontificia Universidad Javeriana, 233 p. 
Robbins, Donald y Ruiz, Édison (2007). Informality and Unemployment: The Role of Public Goods, Payroll Taxes and Penalties. Theory and Simulations for Colombia. Trabajo no publicado. Universidad de Antioquia: Medellín, Colombia, 38 p.

Rodríguez, Gloria y Calderón, Mayda (2015). La economía informal y el desempleo: el caso de la ciudad de Bucaramanga (Colombia). En: Revista Innovar, Universidad Nacional de Colombia,vol. 25, n. ${ }^{\circ} 5$ enero-marzo, p. $41-\overline{58}$.

Rosales, Lucia (2003). Reseña sobre la economía informal y su organización en América Latina. Manchester, Reino Unido: Global Labour Institute, 24 p.

Rosenhouse, Sandra (1988). Identifying the Poor: Is Headship Useful Concept. Living Standards Measurement Study Working Paper. Washington, Estados Unidos de América: Banco Mundial, n. ${ }^{\circ} 58,62 \mathrm{p}$.

Salas, Carlos (2006). El sector informal: auxilio u obstáculo para el conocimiento de la realidad social en América Latina. En: De la Garza Toledo et al. (eds.). Teorías sociales y estudios del trabajo: nuevos enfoques. Ciudad de México, México: UAM, p. 130-148.

Salles, Vania y Tuirán Rodolfo (1999). ¿Cargan las mujeres con el peso de la pobreza? Puntos de vista de un debate. Ciudad de Guatemala, Guatemala: PNUD, 30 p.

Sánchez, Roberto (2013). Enfoques, conceptos y metodologías de medición de la informalidad laboral en Colombia. En: Lecturas de Economía, n. 79,julio-diciembre, p. 9-43.

Schneider, Friedrich (2004). The Size of the Shadow Economies of 145 Countries all over the World: Estimation Results over the Period 1999 to 2003. En: IZA Discussion Paper Series, n. ${ }^{\circ} 1431$, p. 3-58.

Sethuraman, S. V. (1981). The Urban Informal Sector in Developing Countries: Employment, Poverty and Environment (n. ${ }^{\circ}$ XII). Ginebra, Suiza: OIT, 225 p.

Sethuraman, S. V. (1976) El sector urbano no formal: definición, medición y política. En: Revista Internacional del Trabajo, vol. 94, n. ${ }^{\circ}$ 1, p. 77-90.

Stoller, Robert (1985). Presentations of Gender (n. 3). New Haven, Estados Unidos de América: Yale University Press, 230 p.

Tokman, Victor (1992). Beyond Regulation. The Informal Economy in Latin America. Colorado, Estados Unidos de América: Lynne Rienner Pub., 350 p.

Williams, Colin (2015). The informal economy as a path to expanding opportunities. Report prepared for Centre for Development and Enterprise. Sheffield, Reino Unido: University of Sheffield, $44 \mathrm{p}$.

World Trade Organization (2009). Globalization and informal jobs in developing countries. Ginebra, Suiza: International Labour Organization y World Trade Organization, 192 p.

Young, Kate (1991). Reflexiones sobre cómo enfrentar las necesidades de las mujeres. En: Virginia Guzmán, Patricia Portocarrero y Virginia Vargas (eds.). Una nueva lectura: género en el Desarrollo. Lima, Perú: Ediciones Flora Tristán, p. 15-54. 